\title{
An exploratory study of perinatal hair cortisol concentrations in mother-infant dyads with severe psychiatric disorders versus healthy controls
}

Carlinde W. Broeks, Vandhana Choenni, Rianne Kok, Bibian van der Voorn, Ineke de Kruijff, Erica L.T. van den Akker, Elisabeth F.C. van Rossum, Witte J.G. Hoogendijk, Manon H.J. Hillegers, Astrid M. Kamperman and Mijke P. Lambregtse-Van den Berg

\section{Background}

Maternal psychopathology during pregnancy is associated with negative outcomes in offspring. Increased placental transfer of maternal cortisol may contribute to mediate this association. Hair cortisol concentrations (HCCS) appear to be a good biomarker of long-term prenatal stress exposure. Little is known about the associations between severe maternal psychopathology and perinatal infant HCCs.

\section{Aims}

We assessed HCCs in the perinatal period in mother-infant dyads with and without severe psychiatric disorders.

\section{Method}

We examined group differences in HCCs of mother-infant dyads $(n=18)$ subjected to severe maternal psychiatric disorders versus healthy control dyads $(n=27)$. We assessed the correlation of HCCs between mother and infant within both groups, and the association between current maternal symptoms and HCCs in patient dyads.

\section{Results}

Median (interquartile range) and distribution of HCC differed in patients compared with control mothers $(U=468.5, P=0.03)$ HCCS in infants of patients did not differ from control infants $(U=250.0, P=0.67)$. Subsequently, we found that HCCs within healthy control dyads were correlated $(n=27, r 0.55(0.14)$,
$P=0.003)$, but were not within patient dyads $(n=18, r 0.082$

$(0.13), P=0.746)$. HCCs in infants of patients showed a positive correlation with maternal symptoms $(n=16, r=0.63(0.06)$, $P=0.008)$.

\section{Conclusions}

These preliminary findings suggest that infant $\mathrm{HCC}$ reflect perinatal stress exposure. In infants, these early differences could influence lifetime hypothalamic-pituitary-adrenal axis functioning, which might be associated with increased susceptibility to later disease.

\section{Keywords}

Stress exposure; glucocorticoid levels; psychopathology; pregnancy; infant.

\section{Copyright and usage}

(c) The Author(s), 2021. Published by Cambridge University Press on behalf of the Royal College of Psychiatrists. This is an Open Access article, distributed under the terms of the creative Commons Attribution-NonCommercial-ShareAlike licence (http://creativecommons.org/licenses/by-nc-sa/4.0/), which permits non-commercial re-use, distribution, and reproduction in any medium, provided the same Creative Commons licence is included and the original work is properly cited. The written permission of Cambridge University Press must be obtained for commercial re-use.
Children of mothers with psychiatric disorders during pregnancy are at high risk for developing physical and psychiatric disorder later in life. ${ }^{1,2}$ Maternal psychopathology and stress during pregnancy are among the most common intrauterine exposures associated with negative outcomes in offspring, with prevalence rates of $10-15 \%$ for depression and anxiety disorders. ${ }^{3-5}$ Psychiatric disorders are associated with alterations in basal cortisol levels and disturbed variability of the stress response owing to dysregulation of the hypothalamic-pituitary-adrenal (HPA) axis. ${ }^{6,7}$ Accumulating research suggests placental transfer of maternal cortisol might play a mediating role in the effects of maternal psychopathology on the neurocognitive and physical development of the foetus, and increased prenatal maternal cortisol levels have been repeatedly linked to adverse child outcomes in the short and long term, such as lower birth weight, small for gestational age, intellectual disability and behavioural problems. ${ }^{9-11}$ As most previous research has focused on less-affected individuals, the specific aim of this study is to explore the effect of severe and long-lasting psychiatric disorders during pregnancy on early hair cortisol concentrations (HCCs) in mother-infant dyads.

Hair cortisol is a reliable biomarker reflecting chronic systemic cortisol levels, ${ }^{12,13}$ as well as stress exposure. ${ }^{14,15}$ Maternal HCC at
6 weeks' postpartum probably reliably reflects cortisol exposure in the preceding 3 months, which allows us to quantify cortisol exposure from the last 6 weeks of pregnancy to the first 6 weeks' postpartum. ${ }^{16,17}$ In very young infants, less is known about the exact timeframe of exposure, but supposedly HCC reflects cortisol exposure in the intrauterine milieu during late pregnancy and early postpartum cortisol exposure. ${ }^{18}$

\section{HPA axis and psychiatric disorders}

The relationship between psychopathology and HPA axis deviations in general has not been fully elucidated. Specific psychiatric diagnoses, such as major depressive disorder, bipolar disorder and schizophrenia, have been linked to higher basal cortisol levels, whereas anxiety disorders have been associated with a combined profile of higher levels of cortisol during acute stress and lower baseline cortisol levels. ${ }^{19,20}$ The latter finding of lower baseline cortisol levels has also been found in studies on borderline personality disorder. ${ }^{21}$ Post-traumatic stress disorder has been generally linked to lower cortisol levels; however, cortisol appears to be elevated when the traumatic event has happened more recently, or when traumatic 
circumstances are still present. ${ }^{22,23}$ It has been proposed there is a non-linear, two-stage timeline with regard to cortisol dysfunction in relation to trauma: the severity of traumatisation and more temporally distant traumatisation were related to lower HCC, whereas higher HCC was found in more recently traumatised individuals. ${ }^{24}$ Similarly, in depression, it has been found that recurring episodes are associated with lower HCC. This evidence leads to the hypothesis that chronic overactivation of the stress response leads to blunted HPA axis activity over time, indicating that the severity and duration of stress activity might be a more important determinant of basal cortisol levels in patients with severe and long-lasting psychiatric disorders than the nature of the psychiatric diagnosis. ${ }^{25,26}$

\section{Maternal HPA axis functioning and its influence on the foetus}

Altered HPA axis activity in mothers who suffer from severe psychiatric disorders can influence the foetus through intrauterine programming of the HPA axis. ${ }^{19,27}$ There is some evidence that these early alterations in HPA axis functioning contribute to vulnerability to psychiatric disease in offspring later in life, ${ }^{28}$ by early fine-tuning of the HPA axis set point.

Under normal conditions, in the absence of psychopathology or severe stress, maternal and infant cortisol and cortisol responses appear to be positively correlated shortly after birth. ${ }^{29,30}$ This finding is mainly based on research with saliva cortisol, but has been confirmed in animal studies on hair cortisol $^{31}$ and in studies on healthy mother-infant dyads. ${ }^{17}$

Studies evaluating the effects of stress and psychopathology on maternal and foetal HCC during pregnancy and beyond are rapidly emerging, but results are inconclusive. ${ }^{32-38}$ This might be partly explained by differences in study sample (i.e. healthy versus depressed mothers) and different definitions of 'stress' (i.e. perceived stress versus psychiatric symptom scales). Inconsistent results have emerged on the association between maternal prenatal cortisol levels and self-reports of prenatal psychological distress, elevated symptoms of prenatal depression, anxiety and antidepressant use. Evidence in different studies does show that excess maternal cortisol during pregnancy is associated with decreased infant cortisol levels, as measured in infant hair, shortly after birth ${ }^{33,39}$ and at 12 months' postpartum. ${ }^{34}$ In 2-year-old children, Bryson et $\mathrm{al}^{40}$ found a significant association between maternal and infant HCC that was not mediated by measures of early childhood adversity. Furthermore, two studies found that elevated maternal HCC during pregnancy mediated disrupted mother-child interaction in early infancy. ${ }^{26,41}$ These results suggest that maternal stress (i.e. psychiatric symptoms) is inconsistently related to maternal and/or infant HCC, but independently, maternal HCC seems to influence infant cortisol and mother-child interaction both in the early postpartum period and beyond.

The underlying mechanism of transmission of maternal psychopathology during pregnancy through cortisol remains unclear, as cortisol attunement between mother and foetus is composed of complex intrauterine interactions between the maternal, placental and foetal endocrine systems. The placental barrier is not completely impenetrable for transfer of cortisol, as a small proportion (10-20\%) of maternal cortisol does reach the foetus. ${ }^{42}$ However, in stressful situations, more cortisol can cross the placental barrier. ${ }^{43,44}$ Thus, it has been proposed that in stressed mothers, the excess of maternal cortisol levels leads to downregulation of cortisol production in the foetal adrenal, ${ }^{33}$ altering the set point of HPA axis functioning in the foetus. Because psychiatric disorders are associated with altered HPA axis activity, and this is associated with suboptimal HPA axis functioning of the infant, more insight into this process is needed in clinical and healthy dyads, to understand contributing factors and ultimately prevent adverse outcomes for offspring.

\section{Hypotheses}

In the current study, we assessed the association between severe and long-lasting psychiatric disorders and HCCs of mothers and infants. We compared HCCs of patient dyads to healthy control dyads at 6 weeks' postpartum, to further elucidate mechanisms associated with the transgenerational transmission of psychopathology. In accordance with previous studies demonstrating that psychiatric disorders are associated with differential HPA axis disturbances, we expected larger variance in cortisol concentrations in our patient group than in controls. Subsequently, we assessed the effect of the severity of current maternal symptoms on infant HCC.

Also, we expected infant HCC to be associated with maternal perinatal HCC in healthy dyads. Previous research shows that in healthy mother-infant dyads, maternal and infant HCC appear to be positively correlated. Therefore, we expected to find an attuned association of HCC in control dyads. Because maternal psychopathology is associated with both increased and decreased maternal cortisol levels, influenced by the nature, chronicity and genetic heritability of the psychiatric disorder, we expected to find a divergence of this pattern in mothers and infants who were subject to maternal severe psychiatric disorders.

\section{Method}

\section{Study procedure and design}

The current study was embedded in an observational study on parenting capacity of mothers with severe psychiatric disorders and their infant's cognitive and socio-emotional development (the Infant Caregiving Assessment Scales (INCAS) study). All mothers fulfilled criteria for a current severe psychiatric disorder. A common definition of severe psychiatric disorders, or 'severe mental illness', consists of having any psychiatric diagnosis with a treatment duration of 2 years or more, together with dysfunction, as indicated by lower scores on the Global Assessment of Functioning scale. ${ }^{45}$ Common disorders that are referred to are schizophrenia, mood disorders (chronic depression, bipolar disorder), chronic anxiety and personality disorders. ${ }^{46}$

During pregnancy, mothers with severe psychiatric disorders were recruited from specialised psychiatry-obstetrics-paediatric secondary and tertiary out-patient clinics and other specialised mental healthcare institutions where pregnant women who suffer from psychiatric disorders are treated. Healthy control mothers, without current or a history of psychiatric symptoms, were recruited during pregnancy at midwifery practices in the central western part of the Netherlands, consisting primarily of the four largest Dutch cities and their surrounding areas.

The INCAS study was approved by the Erasmus University Medical Center Medical Research Ethics Committee (approval number NL42662.078.12); written informed consent was obtained from all mothers for their own and their infant's participation, and from fathers with legal guardianship.

\section{Exclusion criteria}

In the current study, exclusion criteria for both groups were insufficient amount of hair necessary for cortisol analysis; use of locally administered and systemic corticosteroids during or after pregnancy; use of illicit drugs or alcohol in the last trimester of pregnancy; and perinatal complications, including prematurity. Additionally, control dyads were excluded from analysis when 
maternal global score on the Brief Symptom Inventory (BSI) was in the clinical range or when mothers used psychotropic medication. ${ }^{47}$

\section{Inclusion of clinical sample}

From June 2013 to January 2016, patients and control mothers were included in the INCAS study during their third trimester of pregnancy $(N=129)$. A total of $64 \%$ of participating mothers $(n=83)$ agreed on hair donation at $46 \pm 8.5$ days' postpartum (range $34-84$ days) for themselves, and $45 \%$ for their infant $(n=58)$. After exclusion based on the aforementioned exclusion criteria, HCCs were available for a total of 73 mothers (patient $n=33$, control $n=40$ ) and 47 infants (infant of patient $n=20$, control infant $n=27$ ) (see flowchart in Appendix).

Non-response analyses, comparing mothers and infants who did and did not donate hair for cortisol measurement, showed no differences with regards to maternal age, ethnicity, educational level, psychiatric symptoms, and infant birth weight or gestational age.

\section{Measures}

HCCS

Mother and child HCCs were determined from hair strands collected 6 weeks' postpartum (46 days \pm 8.5 , range $34-84$ days). All samples were collected according to researcher protocol. In adults, scalp hair has a predictable growth rate of approximately $1 \mathrm{~cm}$ per month, making it possible to have an estimate of long-term exposure to cortisol. ${ }^{48,49}$ When collected at 6 weeks' postpartum, HCC in the proximal $3 \mathrm{~cm}$ of maternal hair reflects the maternal HPA axis activity over the first 6 weeks after childbirth and the last 6 weeks of pregnancy. ${ }^{16}$

A small strand of hair was cut from the posterior vertex of the scalp, as close as possible to the scalp. Hair strands were taped to a piece of paper with the scalp end marked, and stored in an envelope at room temperature until further analysis. The proximal $3 \mathrm{~cm}$ of maternal hair samples were weighed and minced. For infants, the full length of the hair was analysed with a minimum of $1.25 \mathrm{mg}$, for reliable measurement. For extraction of cortisol, LC-grade methanol was used at $25^{\circ} \mathrm{C}$, for $18 \mathrm{~h}$, in the presence of labelled glucocorticoids as internal standard. The extraction was centrifuged and cleaned. Cortisol concentrations were quantified by liquid chromatography with tandem mass spectrometry (Waters XEVO-TQ-S system; Waters Corporation, Milford, MA, USA). Measurements were reported in picograms per milligram of hair, and log-transformed $(10 \log )$ to approach normality. ${ }^{50}$

\section{Psychiatric diagnosis and current symptoms}

Presence and history of psychiatric diagnoses were examined with the Structured Clinical Interview for DSM-IV Axis I Disorders (SCID-I) and Structured Clinical Interview for DSM-IV Axis II Disorders (SCID-II), by a trained interviewer. ${ }^{51,52}$ SCID-I and SCID-II are considered to be the gold standard of semi-structured assessment instruments for clinical psychiatric disorders, with adequate to excellent validity and interrater reliability. ${ }^{53}$

Level of current symptoms in both the patient and control group were measured with the BSI, at 6 weeks' postpartum. ${ }^{54}$ Severity of stress was indicated by the Global Severity Index (GSI). ${ }^{55}$ The BSI comprises 53 items on nine symptom dimensions (somatisation, obsession-compulsion, interpersonal sensitivity, depression, anxiety, hostility, phobic anxiety, paranoid ideation and psychoticism). The GSI presents the mean BSI score. Normative data are available for clinical and non-clinical samples. The BSI has a high internal consistency, moderate test-retest reliability and strong convergent validity with measures of emotional functioning. ${ }^{47}$ In our sample, Cronbach's alpha was 0.93 in patients and 0.82 in controls.

\section{Covariates and potential confounders}

Demographic data; information on smoking, alcohol and illicit drug use; and exposure to psychotropic medication during pregnancy were collected during the third trimester of pregnancy (patients) and at 6 weeks' postpartum (controls), using self-reports. Confounders were selected $a$ priori, based on previous research. ${ }^{56,57}$ The following confounders were controlled for the following: child gender, gestational age and birth weight; and maternal age, ethnicity, socioeconomic status, parity (primiparity versus multiparity), tobacco use and use of psychotropic medication.

\section{Data analyses}

Demographic and clinical characteristics of the control and patient sample are reported, and differences between the samples were tested with $\chi^{2}$-tests (for categorical variables) and $t$-tests or MannWhitney $U$-tests (for continuous variables). Differences in HCCs between patient and control mothers, for diagnostic subgroups in the patient group, and between infants, were tested using MannWhitney $U$-tests. For this purpose, HCCs were log-transformed.

We estimated the association between maternal and infant HCC in both the patient and control sample by regression analysis. Preliminary analyses did not show significant correlations between hair characteristics (e.g. hair treatment in the past 3 months, heavy transpiration, hair product use before hair collection) in mothers $(P>0.201)$ or infants $(P>0.577)$, or between timing of the hair sample (range 34-84 days' postpartum) and infant HCC $(P=0.770)$; accordingly, we did not control for these variables in the regression analyses. To adjust for the effects of other potential confounders, we calculated a propensity score including all available confounders as summarised in subheading 'Covariates and potential confounders', and included the propensity score as a single covariate in all analyses. ${ }^{58}$ Differences in maternalinfant HCC associations between the patient and control samples were tested with Fisher $z$-scores.

We also explored whether maternal symptom severity was related to HCC in mothers and infants. Therefore, we estimated the association between perinatal symptom severity levels (based on BSI scores) and maternal and infant HCCs, using regression analysis. We conducted a sensitivity analysis, repeating the regression analysis but leaving out two outliers.

Results from the regression analyses are reported as correlation coefficient $(r)$ and s.e. ${ }^{59} \mathrm{Q}-\mathrm{Q}$ plots were used to check all data for normality of the distribution. HCC data were checked for extreme outliers (defined as below quartile 1 (Q1) - 1.5 interquartile range (IQR) or above quartile $3(\mathrm{Q} 3)+1.5$ interquartile range (IQR)), which were removed from all analyses $(n=4)$. Statistical analyses were performed with SPSS version 24 for Windows (IBM, New York, USA).

\section{Results}

\section{Background and clinical characteristics}

A sample description is displayed in Tables 1 and 2. Mothers in the patient and control group did not differ with regard to age and ethnicity. Lower educational level and smoking were more common among patients. Expectedly, infants of patients had a significantly lower gestational age and birth weight compared with control infants. ${ }^{46,60}$ In the patient group, depressive and anxiety disorders were most common (33.3 and $51.1 \%$, respectively), followed by bipolar disorders (18.2\%). A considerable percentage of mothers 
Table 1 Demographic characteristics of patients $(n=33)$, control mothers $(n=40)$, infants of patients $(n=20)$ and control infants $(n=27)$

\begin{tabular}{|c|c|c|c|}
\hline & $\begin{array}{l}\text { Patient } \\
\text { group }\end{array}$ & $\begin{array}{l}\text { Control } \\
\text { group }\end{array}$ & $P$-valt \\
\hline \multicolumn{4}{|l|}{ Demographic characteristics } \\
\hline Maternal age, years, mean (s.d.) & $32.2(6.0)$ & $31.9(4.4)$ & 0.12 \\
\hline Maternal ethnicity, White, $n$ (\%) & $27(81.8)$ & $32(88.9)$ & 0.41 \\
\hline Education level, low, n (\%) & $27(84.4)$ & $16(45.7)$ & 0.001 \\
\hline Tobacco use & $11(33.3)$ & $3(8.3)$ & 0.008 \\
\hline Gestational age, weeks, mean (s.d.) & $38.3(1.9)$ & $39.7(1.3)$ & 0.002 \\
\hline $\begin{array}{l}\text { Infant birth weight, grams, } \\
\text { mean (s.d.) }\end{array}$ & $3227(456)$ & $3652(608)$ & 0.002 \\
\hline Infant gender, male, $n$ (\%) & $13(66.7)$ & $18(45.0)$ & 0.06 \\
\hline \multicolumn{4}{|l|}{ Maternal symptom severity } \\
\hline $\begin{array}{l}\text { Brief Symptom Inventory, mean GSI } \\
\text { score (s.d.) }\end{array}$ & $0.95(0.66)^{\mathrm{a}}$ & $0.21(0.23)^{\mathrm{a}}$ & $<0.001$ \\
\hline \multicolumn{4}{|c|}{$\begin{array}{l}\text { GSI, Global Severity Index. } \\
\text { a. Mean reference range was } 0.93-1.32 \text { for Dutch clinical females and } 0.29-0.45 \text { for } \\
\text { healthy females. }\end{array}$} \\
\hline
\end{tabular}

(39.4\%) had two or more Axis I disorders (e.g. depressive disorder and panic disorder). Furthermore, half of the patients had a comorbid personality disorder (48.4\%), mostly in Cluster C (avoidant, dependent or obsessive-compulsive personality disorder). Approximately two-thirds (67.7\%) of the patient group used psychotropic medication during pregnancy, which were mostly antidepressants, followed by antipsychotics and hypnotics. A smaller group of mothers used two or more psychotropic medications (19.4\%).

\section{Differences in HCCs between patient and control dyads}

HCC of patient and control dyads are displayed in Fig. 1. Median (interquartile range) and distribution of HCC were significantly different in patients compared with control mothers $(U=468.5, P=0.03)$. Results did not differ in infants of patients $(U=250.0, P=0.67)$.

\section{Correlation of HCCs within clinical and control mother-infant dyads}

We found a positive correlation between maternal perinatal HCC and infant HCC in the control group $(n=27, r=0.55(0.14)$, $P=0.003)$. The correlation between maternal perinatal HCC and infant HCC in the patient group was non-significant $(n=18$, $r=0.082$ (0.13), $P=0.746$; see Fig. 2). The correlations between maternal perinatal HCC and infant HCC were significantly different across the patient and control group $(z=-1.64, P=0.05)$. The correlation analyses were repeated with the propensity score, to adjust for confounders. After adjustment, the strength of the correlation between HCCs in control mother-infant dyads increased somewhat $(r=0.65 \quad(0.13), P=0.001)$. In patient mother-infant dyads, the correlation increased greatly, but remained non-significant $(r=0.37(0.13), P=0.16)$.

\section{Correlation of current maternal symptom severity with HCCs in patient dyads}

We explored if maternal symptom severity in the perinatal period is correlated with maternal and infant HCC. Results are displayed in Fig. 3.

In mothers, symptom severity was not correlated with HCC ( $n$ $=23, r=-0.09(0.12), P=0.67)$. In infants, a positive correlation between maternal perinatal symptom severity and HCC was found ( $n=16, r=0.63(0.06), P=0.008)$. The correlation analyses were repeated with the propensity score, to adjust for confounders. After adjustment, the strength of the correlation between symptom severity and maternal perinatal HCC ( $r=0.08(0.13), P=0.70)$ and infant HCC $(r=0.59(0.18), P=0.02)$ remained in a similar range.

$\begin{array}{lc}\text { Table } 2 \text { Clinical characteristics of patients }(n=33) & \\ \text { Maternal psychiatric characteristics } & N(\%) \\ \text { Axis I psychiatric disorder } & \\ \quad \text { Depressive disorder, } n \text { (\%) } & 11(33.3) \\ \text { Anxiety disorder, } n \text { (\%) } & 17(51.1) \\ \text { Psychotic disorder, } n \text { (\%) } & 2(6.1) \\ \text { Bipolar disorder, } n \text { (\%) } & 6(18.2) \\ \text { Comorbidity ( } 2 \text { Axis I disorders), } n(\%) & 13(39.4) \\ \text { Axis II personality disorder } & \\ \text { Cluster A, } n \text { (\%) } & 5(16.1) \\ \text { Cluster B, } n \text { (\%) } & 3(9.7) \\ \text { Cluster C, } n \text { (\%) } & 10(32.3) \\ \text { No personality disorder, } n \text { (\%) } & 16(51.6) \\ \text { Psychotropic medication use }{ }^{a} & \\ \text { SSRI/SNRI/TCA, } n \text { (\%) } & 14(45.2) \\ \text { Antipsychotics, } n \text { (\%) } & 8(25.8) \\ \text { Hypnotics/anxiolytics, } n \text { (\%) } & 3(9.1) \\ \geq 2 \text { psychotropic medications, } n \text { (\%) } & 6(19.4) \\ \text { No psychotropic medication use, } n \text { (\%) } & 10(32.3) \\ \text { SSRI, Selective Serotonin Reuptake Inhibitor; SNRI, Selective Serotonin and Noradrenalin } \\ \text { Reuptake Inhibitor; TCA, Tricyclic Antidepressants. } \\ \text { a. Any exposure during pregnancy. }\end{array}$

In the sensitivity analysis, leaving out the two outlier infants on the right, the strength of the correlation between maternal symptom severity and infant HCC remained in a similar range as our original result.

\section{Discussion}

In this study, we explored the influence of severe psychiatric disorders on HCCs of mothers and newborn infants. We found a significantly wider range of HCCs in mothers with severe psychiatric disorders compared with controls, but we did not find differences in infants. We also found that HCC of patients and infants of patients were not associated, whereas in control dyads, we found a significant positive association between mother and child HCCs. In infants of patients, HCCs were positively associated with maternal symptom severity.

\section{HCCs in patient and control groups}

In our study, a significant variation in HCC was found in mothers with psychopathology, showing both (mainly) higher and lower values than control mothers. One explanation for this finding is the nature of our clinical sample, in which women with severe and long-lasting psychiatric disorders were selected. Previous studies have shown cortisol levels in patients with mood disorders change over time. First episodes are more often associated with higher cortisol levels, whereas long-term duration and recurrence of episodes might diminish the sensitivity of the HPA axis over time. ${ }^{6,61}$ Heterogeneity of psychiatric diagnoses and high prevalence of medication use might also be critical factors in differences of HPA axis functioning and long-term release of cortisol in affected mothers. ${ }^{19,62}$

Infants of patients showed no significant differences with regard to variation in HCC compared with control infants. Previous studies have shown that higher maternal perinatal HCC predicted lower HCC in newborn infants early postpartum. ${ }^{33,39}$ We could not replicate this finding. Three factors may contribute to this inconsistence. First, the sample size of our study might not have been sufficient to uncover differences in our patient dyads. Second, the mothers in the aforementioned studies were healthy or subject to mood and anxiety disorders and only used antidepressants, whereas patients in this study had high rates of comorbidity and 

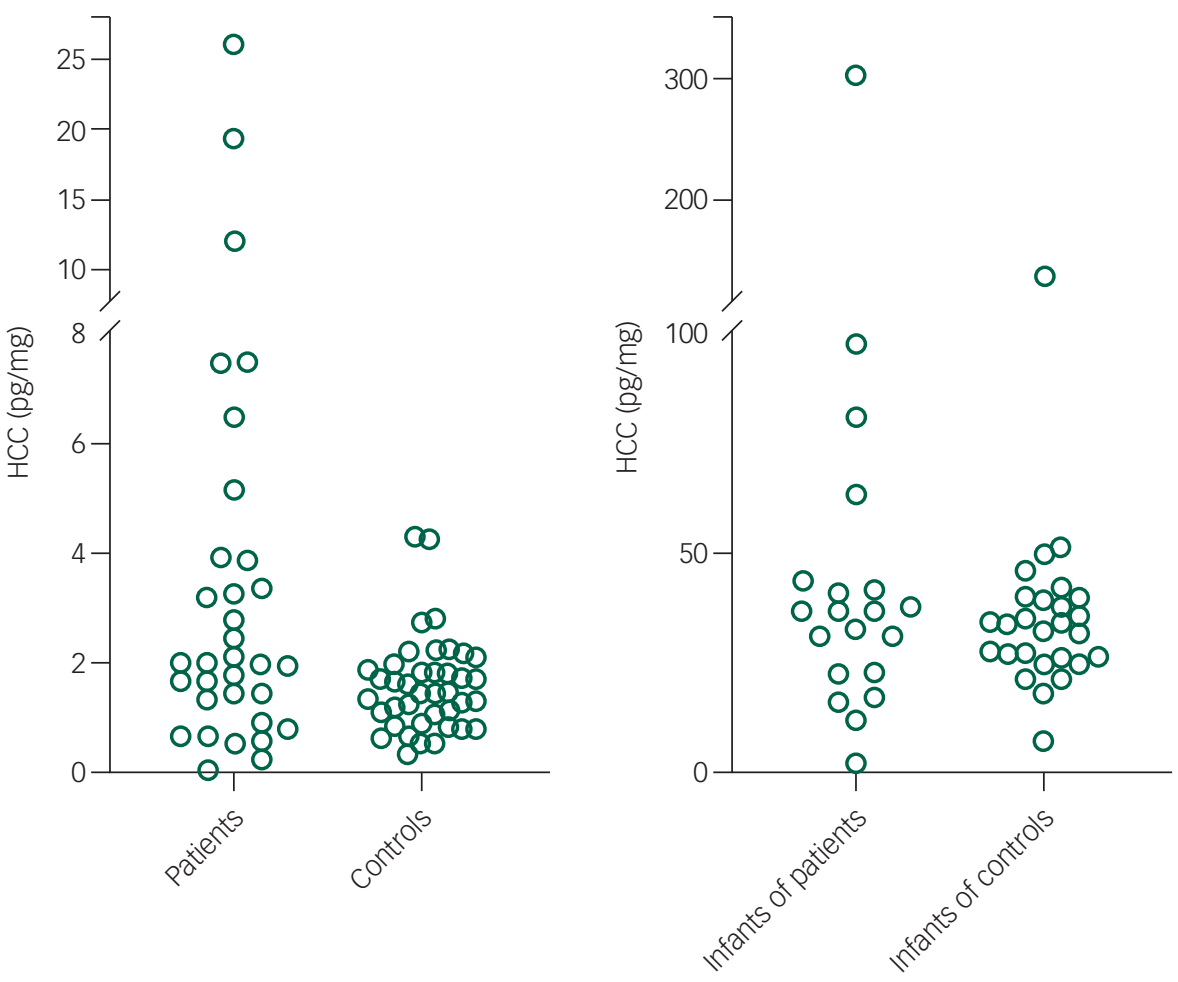

Fig. 1 Distribution of hair cortisol concentrations (HCCS) in patient versus control mothers, and infants of patients versus infants of controls. Median (interquartile range) and distribution of HCCs were significantly different in patients compared with control mothers $(U=468.5, P=0.03)$. Results did not differ in infants of patient $(U=250.0, P=0.67)$.

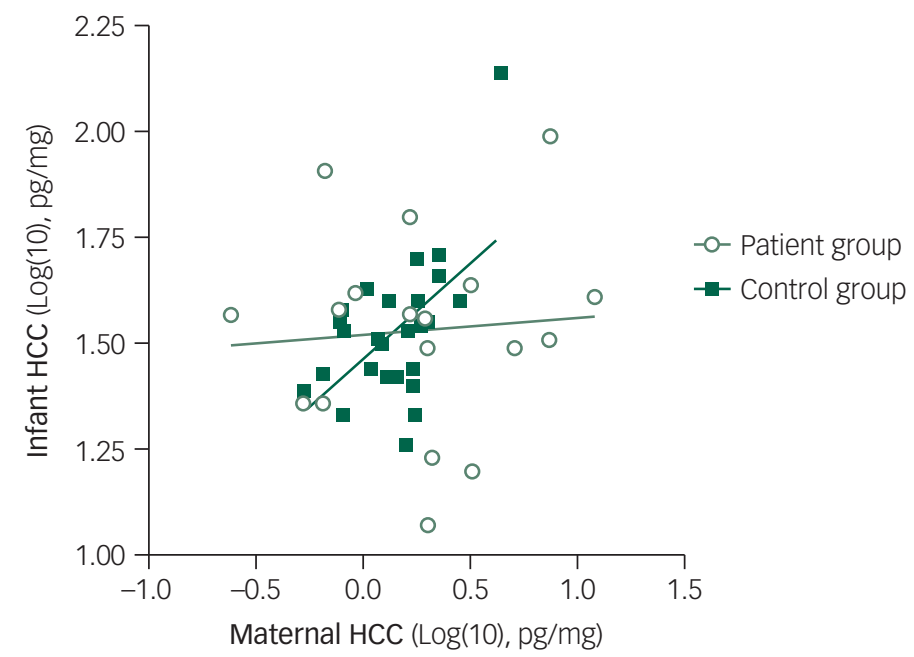

Fig. 2 Correlation between mother and infant log-transformed hair cortisol concentrations (HCCs). Figure based on unadjusted results.

other medication use (including antipsychotics). Third, the absence of marked differences of HCC in infants of the patient group might reflect that foetal exposure to increased or decreased maternal cortisol concentrations during pregnancy is effectively regulated by the dynamic nature of placental $11 \beta$-hydroxysteroid dehydrogenase type II (11 -HSD-2). ${ }^{63}$

\section{Prenatal synchrony of HCCs between mother and child}

In line with previous studies, ${ }^{17,39}$ we found a positive association between maternal and infant HCC in healthy control dyads. This finding might reflect early physiological synchrony, which is defined as the matching of biological states between mother and child that develops via interactions among genetic predispositions, prenatal programming and postnatal behaviour. ${ }^{64,65}$ In motherinfant dyads subject to severe psychiatric disorders, we found a divergence of this pattern. This might indicate that synchrony of the HPA axis between mother and child might be prenatally affected by the presence of a maternal psychiatric disorder. This finding should be interpreted with caution because the two groups in this study differed with regards to relevant demographic and obstetric variables (e.g. lower education level in patients, lower birth weight 

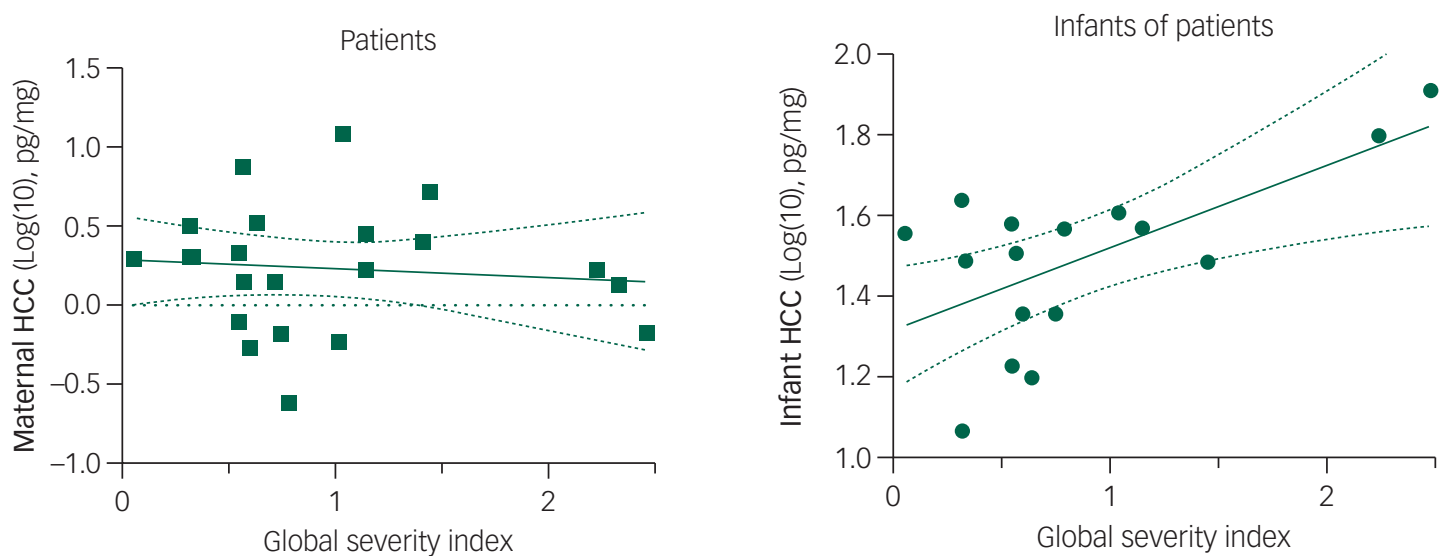

Fig. 3 Association between maternal symptom severity by means of the Global Severity Index and log-transformed hair cortisol concentrations (HCCS) of patients (left) and infants of patients (right). Figure based on unadjusted results.

and gestational age in infants of patients), but it should also be noted that the findings remained the same when these factors were controlled for.

\section{HCCs and (self-reported) symptom severity}

We did not find a correlation between maternal perinatal HCC and self-reported symptom severity at 6 weeks' postpartum. Because of the previously mentioned blunted cortisol responses in long-lasting psychopathology, the absence of an association between HCC and maternal-reported stress levels might indicate reduced responsiveness of the HPA axis to stressful experiences. ${ }^{7}$ However, the relationship between the human concept of stress and HPA axis functioning is an ongoing subject of debate. A recent meta-analysis of Kalliokoski et al ${ }^{66}$ on hair glucocorticoids as a measure of stress suggests that self-reported assessments of stress poorly correlate with HPA axis functioning. Furthermore, symptom assessments in our study were obtained postpartum, which is an especially chaotic transition in a woman's life. It might not be accurate to relate this to cortisol levels that presumably reflect third trimester exposure of cortisol. It also cannot be ruled out that other physical factors that are important determinants of cortisol levels, such as obesity, metabolic syndrome and cardiovascular disease, were overrepresented in the patient group compared with the control group, and therefore may have influenced the outcomes. ${ }^{67,68}$

Interestingly, in infants of patients, we found that higher maternal symptom severity was associated with higher infant HCC, after statistically controlling for known covariates of HCC. There are several possible explanations for this finding. It has been proposed that the placental barrier function, inactivating cortisol by $11 \beta-$ HSD-2, may be impaired by stress, ${ }^{44,69}$ allowing an increased passage of maternal cortisol to the foetus. ${ }^{63}$ Also the production of placental corticotropin-releasing hormone $(\mathrm{CRH})$ might be reacting to blunting of the maternal HPA axis, leading to stimulation of the foetal adrenal. ${ }^{70}$ Stressful circumstances during delivery and in the postpartum period might also contribute to higher infant HCC. However, this finding is subject to the same limitations (i.e. maternal self-reported stress) as in mothers, and has to be interpreted with caution.

\section{Strengths and limitations}

Our study has several strengths and limitations. The foremost strength of this study is our patient sample of mothers with various severe psychiatric disorders, representing the heterogeneity of clinical populations. Further strengths are the non-invasive measurement of chronic stress in hair performed with the stateof-the-art liquid chromatography with tandem mass spectrometry method, and the availability of detailed and reliable diagnostic information, as well as the possibility to adjust for various covariates. Limitations include the limited sample size of subgroups, which only allowed for an initial exploration. Additionally, we only measured psychiatric symptom severity at 6 weeks' postpartum, and could therefore not take into account the possible variation of symptoms over time.

\section{Conclusions and future research}

In the current study, we observed differences in the association between HCCs of patients and their infants compared with healthy controls and their infants. Where in healthy control dyads there seems to be perinatal synchrony of HPA axis functioning in mother and infant, our findings suggest there is a divergence of this pattern in mother-infant dyads subjected to long-lasting and severe psychiatric disorders. In infants, these early differences might influence lifetime HPA axis functioning, as has been suggested in previous research. ${ }^{28}$ In turn, altered HPA axis functioning may increase susceptibility to disease, both physically and mentally. Future longitudinal studies in larger clinical samples should examine how maternal and infant hair cortisol levels are intertwined perinatally, in the early postpartum period and beyond.

Carlinde W. Broeks $(\mathbb{D}$, Department of Psychiatry, Erasmus University Medical Center the Netherlands; and Department of Psychiatry, Arkin Institute for Mental Health, the Netherlands; Vandhana Choenni (D), Department of Child and Adolescent Psychiatry/ Psychology, Erasmus University Medical Center, the Netherlands; Rianne Kok, Department of Psychology, Education and Child Studies, Erasmus University Rotterdam, the Netherlands; Bibian van der Voorn, Department of Pediatric Endocrinology, Obesity Center CGG, Erasmus MC-Sophia Children's Hospital, the Netherlands; and Division of Endocrinology, Department of Internal Medicine, Erasmus University Medical Center, the Netherlands; Ineke de Kruijff, Department of Pediatrics, St Antonius Hospital Nieuwegein, the Netherlands; EricaL.T. vandenAkker, Department of Pediatrics, Erasmus MC-Sophia Children's Hospital, the Netherlands; Elisabeth F. C. van Rossum, Division of Endocrinology, Department of Internal Medicine, Erasmus University Medical Center, the Netherlands; Witte J.G. Hoogendijk, Department of Psychiatry, Erasmus University Medical Center, the Netherlands; Manon H.J. Hillegers, Department of Child and Adolescent Psychiatry/Psychology, Erasmus University Medical Center, the Netherlands; AstridM. Kamperman, Department of Psychiatry, Erasmus University Medical Center, the Netherlands; and Epidemiological and Social Psychiatric University Medical Center, the Netherlands; and Epidemiological and Social Psyc
Research Institute, Erasmus University Medical Center, the Netherlands; Mijke P. Lambregtse-Van den Berg, Department of Psychiatry, Erasmus University Medical Center, the Netherlands; and Department of Child and Adolescent Psychiatry/ Psychology, Erasmus University Medical Center, the Netherlands

Correspondence: Mijke P. Lambregtse-Van den Berg. Email: mijke.vandenberg@erasmusmc.nl

First received 14 Jan 2020, final revision 26 Aug 2020, accepted 19 Nov 2020 


\section{Data availability}

Data are stored at the institutional database of the Erasmus Medical Center in Rotterdam, The Netherlands. The data-sets on which the analyses are based are available on request to the Local Ethics Committee of the Erasmus Medical Center in Rotterdam. To request the data please contact the corresponding author, M.P.L.-V.d.B., or Dr Joke Tulen (j.h.m.tulen@eras musmc.nl). The data are not publicly available due to ethical restrictions and patient confidentiality requirements.

\section{Author contributions}

C.W. B. contributed to conceptualization, data curation, formal analysis, methodology, writing original draft, writing - review and editing V.C. contributed to conceptualization, data curation, funding acquisition, methodology, writing - review and editing. R.K. contributed to conceptualfunding acquisition, methodology, writing - review and editing. R.K. contributed to conceptual-
ization, data curation, funding acquisition, methodology, writing - review and editing. B.v.d.V. contributed to writing - review and editing. I.d.K. contributed to writing - review and editing.

Appendix: flowchart
E.L.T.V.d.A. contributed to writing - review and editing. E.F.C.V.R. contributed to writing - review and editing. W.J.G.H. contributed to writing - review and editing. M.H.J.H. contributed to writing review and editing. A.M.K. contributed to conceptualization, formal analysis, methodology, writing - review and editing. M.P.L.-V.d.B. contributed to conceptualization, data curation, formal analysis, funding acquisition, methodology, writing - review and editing.

\section{Funding}

This research has been funded by the Sophia Foundation for Scientific Research (grant no. 670).

\section{Declaration of interest}

None.

ICMJE forms are in the supplementary material, available online at https://doi.org/10.1192/ bjo.2020.159.

\section{Appendix: flowchart}

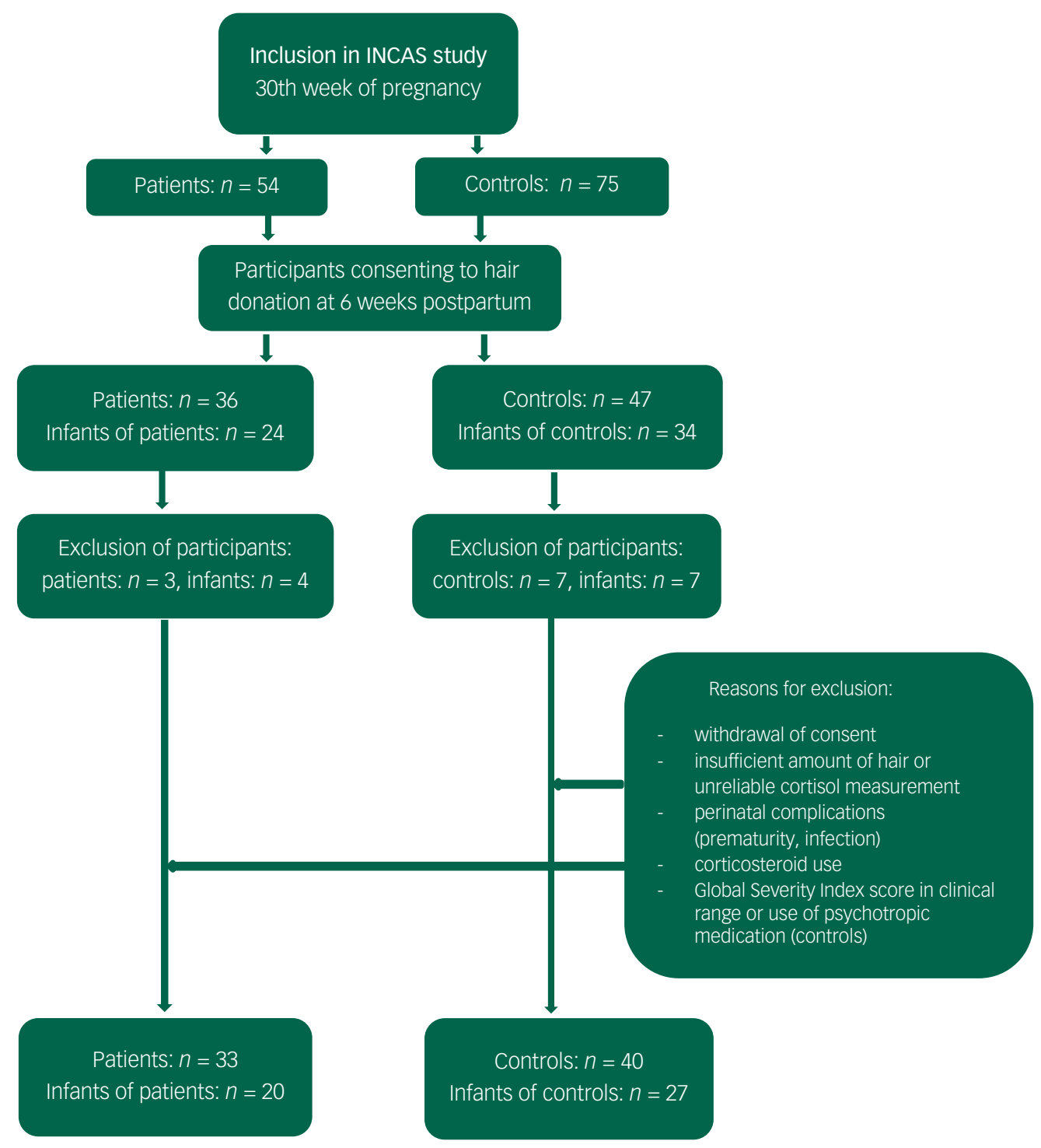

\section{References}

1 Goodman SH, Rouse MH, Connell AM, Broth MR, Hall CM, Heyward D. Maternal depression and child psychopathology: a meta-analytic review. Clin Child Fam Psychol Rev 2011; 14(1): 1-27.

2 Sanger C, Iles JE, Andrew CS, Ramchandani PG. Associations between postnatal maternal depression and psychological outcomes in adolescent offspring: a systematic review. Arch Women's Ment Health 2015; 18(2): 147-62.
3 Bennett HA, Einarson A, Taddio A, Koren G, Einarson TR. Prevalence of depression during pregnancy: systematic review. Obstet Gynecol 2004; 103(4): 698709.

4 Cook N, Ayers S, Horsch A. Maternal posttraumatic stress disorder during the perinatal period and child outcomes: a systematic review. J Affect Disord 2018; 225: 18-31.

5 Dennis CL, Falah-Hassani K, Shiri R. Prevalence of antenatal and postnatal anxiety: systematic review and meta-analysis. Br J Psychiatry 2017; 210(5): 315-23. 
6 Booij SH, Bouma EM, de Jonge P, Ormel J, Oldehinkel AJ. Chronicity of depressive problems and the cortisol response to psychosocial stress in adolescents: the TRAILS study. Psychoneuroendocrinology 2013; 38(5): 659-66.

7 Zorn JV, Schur RR, Boks MP, Kahn RS, Joels M, Vinkers CH. Cortisol stress reactivity across psychiatric disorders: a systematic review and meta-analysis. Psychoneuroendocrinology 2017; 77: 25-36.

8 Monk C, Feng T, Lee S, Krupska I, Champagne FA, Tycko B. Distress during pregnancy: epigenetic regulation of placenta glucocorticoid-related genes and fetal neurobehavior. Am J Psychiatry 2016; 173(7): 705-13.

9 Buitelaar JK, Huizink AC, Mulder EJ, de Medina PGR, Visser GHA. Prenatal stress and cognitive development and temperament in infants. Neurobiol Aging 2003; 24: S53-60.

10 Hantsoo L, Kornfield S, Anguera MC, Epperson CN. Inflammation: a proposed intermediary between maternal stress and offspring neuropsychiatric risk. Biol Psychiatry 2019; 85: 97-106.

11 Talge NM, Neal C, Glover V, the Early Stress, Translational Research and Prevention Science Network: Fetal and Neonatal Experience on Child and Adolescent Mental Health et al. Antenatal maternal stress and long-term effects on child neurodevelopment: how and why? J Child Psychol Psychiatry 2007; 48(3-4): 245-61.

12 Russell E, Kirschbaum C, Laudenslager ML, Stalder T, de Rijke Y, van Rossum EF, et al. Toward standardization of hair cortisol measurement: results of the first international interlaboratory round robin. Ther Drug Monit 2015; 37(1): 71-5.

13 Manenschijn L, Koper JW, Lamberts SW, van Rossum EF. Evaluation of a method to measure long term cortisol levels. Steroids 2011; 76(10-11): 1032-6.

14 Raul JS, Cirimele V, Ludes B, Kintz P. Detection of physiological concentrations of cortisol and cortisone in human hair. Clin Biochem 2004; 37(12): 1105-11.

15 Mustonen P, Karlsson L, Scheinin NM, Kortesluoma S, Coimbra B, Rodrigues AJ, et al. Hair cortisol concentration (HCC) as a measure for prenatal psychological distress - a systematic review. Psychoneuroendocrinology 2018; 92: 21-8.

16 Kirschbaum C, Tietze A, Skoluda N, Dettenborn L. Hair as a retrospective calendar of cortisol production-Increased cortisol incorporation into hair in the third trimester of pregnancy. Psychoneuroendocrinology 2009; 34(1): 32-7.

17 Hollanders JJ, van der Voorn B, Kieviet N, Dolman KM, de Rijke YB, van den Akker ELT, et al. Interpretation of glucocorticoids in neonatal hair: a reflection of intrauterine glucocorticoid regulation? Endocr Connect 2017; 6(8): 692-9.

18 de Kruijff I, Noppe G, Kieviet N, Choenni V, Lambregtse-van den Berg MP, Begijn DGA, et al. LC-MS/MS-based reference intervals for hair cortisol in healthy children. Psychoneuroendocrinology 2020; 112: 104539.

19 Staufenbiel SM, Penninx BW, Spijker AT, Elzinga BM, van Rossum EF. Hair cortisol, stress exposure, and mental health in humans: a systematic review. Psychoneuroendocrinology 2013; 38(8): 1220-35

20 Olff M, Güzelcan Y, de Vries G-J, Assies J, Gersons BPR. HPA- and HPT-axis alterations in chronic posttraumatic stress disorder. Psychoneuroendocrinology 2006 31(10): 1220-30

21 Thomas N, Gurvich C, Hudaib AR, Gavrilidis E, Kulkarni J. Systematic review and meta-analysis of basal cortisol levels in borderline personality disorder compared to non-psychiatric controls. Psychoneuroendocrinology 2018; 102 149-57.

22 Steudte-Schmiedgen S, Kirschbaum C, Alexander N, Stalder T. An integrative model linking traumatization, cortisol dysregulation and posttraumatic stress disorder: insight from recent hair cortisol findings. Neurosci Biobehav Rev 2016; 69: 124-35.

23 van den Heuvel LL, Stalder T, du Plessis S, Suliman S, Kirschbaum C, Seedat S. Hair cortisol levels in posttraumatic stress disorder and metabolic syndrome. Stress 2020; 23: 577-89.

24 Gao W, Zhong P, Xie Q, Wang H, Jin J, Deng H, et al. Temporal features of elevated hair cortisol among earthquake survivors. Psychophysiology 2014; 51 (4): $319-26$

25 Danese A, Moffitt TE, Harrington $H$, Milne BJ, Polanczyk G, Pariante CM, et al Adverse childhood experiences and adult risk factors for age-related disease: depression, inflammation, and clustering of metabolic risk markers. Arch Pediatr Adolesc Med 2009; 163(12): 1135-43.

26 Nystrom-Hansen M, Andersen MS, Khoury JE, Davidsen K, Gumley A, LyonsRuth $\mathrm{K}$, et al. Hair cortisol in the perinatal period mediates association between maternal adversity and disrupted maternal interaction in early infancy. Dev Psychobiol 2019; 61: 543-56.

27 Van den Bergh BRH, van den Heuvel Ml, Lahti M, Braeken M, de Rooij SR, Entringer $\mathrm{S}$, et al. Prenatal developmental origins of behavior and menta health: the influence of maternal stress in pregnancy. Neurosci Biobehav Rev 2020; 117: 26-64

28 Molenaar NM, Tiemeier $\mathrm{H}$, van Rossum EFC, Hillegers MHJ, Bockting CLH, Hoogendijk WJG, et al. Prenatal maternal psychopathology and stress and offspring HPA axis function at 6 years. Psychoneuroendocrinology 2019: 99: 120-7.
29 Sethre-Hofstad L, Stansbury K, Rice MA. Attunement of maternal and child adrenocortical response to child challenge. Psychoneuroendocrinology 2002; 27(6): 731-47.

30 Stenius F, Theorell T, Lilja G, Scheynius A, Alm J, Lindblad F. Comparisons between salivary cortisol levels in six-months-olds and their parents. Psychoneuroendocrinology 2008; 33(3): 352-9.

31 Meise K, von Engelhardt N, Forcada J, Hoffman Jl. Offspring hormones reflect the maternal prenatal social environment: potential for foetal programming? PLOS One 2016; 11(1): e0145352.

32 Orta OR, Tworoger SS., Terry KL, Coull BA, Gelaye B, Kirschbaum C, et al. Stress and hair cortisol concentrations from preconception to the third trimester. Stress 2019; 22(1): 60-9.

33 Van der Voorn B, Hollanders JJ, Kieviet N, Dolman KM, de Rijke YB, van Rossum $\mathrm{EFC}$, et al. Maternal stress during pregnancy is associated with decreased cortisol and cortisone levels in neonatal hair. Horm Res Paediatr 2018; 90: 1-9.

34 Galbally M, van Rossum EFC, Watson SJ, de Kloet ER, Lewis AJ. Trans-generational stress regulation: mother-infant cortisol and maternal mental health across the perinatal period. Psychoneuroendocrinology 2019; 109: 104374.

35 Mustonen P, Karlsson L, Kataja EL, Scheinin NM, Kortesluoma S, Coimbra B, et al. Maternal prenatal hair cortisol is associated with prenatal depressive symptom trajectories. Psychoneuroendocrinology 2019; 109: 104383

36 Conradt E, Shakiba N, Ostlund B, Terrell S, Kaliush P, Shakib JH, et al. Prenatal maternal hair cortisol concentrations are related to maternal prenatal emotion dysregulation but not neurodevelopmental or birth outcomes. Dev Psychobiol 2020; 62(6): 758-67.

37 Alen NV, Hostinar CE, Mahrer NE, Martin SR, Guardino C, Shalowitz MU, et al. Prenatal maternal stress and child hair cortisol four years later: evidence from a low-income sample. Psychoneuroendocrinology 2020; 117: 104707.

38 Kim MY, Kim GU, Son HK. Hair cortisol concentrations as a biological marker of maternal prenatal stress: a systematic review. Int J Environ Res Public Health 2020; 17(11): 4002

39 Romero-Gonzalez B, Caparros-Gonzalez RA, Gonzalez-Perez R, DelgadoPuertas P, Peralta-Ramirez Ml. Newborn infants' hair cortisol levels reflect chronic maternal stress during pregnancy. PLOS One 2018; 13(7): e0200279.

40 Bryson HE, Mensah F, Goldfeld S, Price AMH, Giallo R. Hair cortisol in motherchild dyads: examining the roles of maternal parenting and stress in the context of early childhood adversity. Eur Child Adolesc Psychiatry [Epub ahead of print] 22 Apr 2020. Available from: https://doi.org/10.1007/s00787-020-01537-0.

41 Khoury JE, Bosquet Enlow M, Patwa MC, Lyons-Ruth K. Hair cortisol in pregnancy interacts with maternal depressive symptoms to predict maternal disrupted interaction with her infant at 4 months. Dev Psychobiol 2020; 62: 768-82.

42 Zhu P, Wang W, Zuo R, Sun K. Mechanisms for establishment of the placental glucocorticoid barrier, a guard for life. Cell Mol Life Sci 2019; 76(1): 13-26.

43 Reynolds RM. Glucocorticoid excess and the developmental origins of disease: two decades of testing the hypothesis - 2012 Curt Richter Award Winner. Psychoneuroendocrinology 2013; 38(1): 1-11.

44 Cottrell EC, Seckl JR. Prenatal stress, glucocorticoids and the programming of adult disease. Front Behav Neurosci 2009; 3: 19.

45 Jones SH, Thorncroft G, Coffey M, Dung G. A brief mental health outcome scale reliability and validity of the Global Assessment of Functioning (GAF). $\mathrm{Br} J$ Psychiatry 1995; 166: 654-9.

46 Ruggeri M, Leese M, Thornicroft G, Bisoffi G, Tansella M. Definition and prevalence of severe and persistent mental illness. Br J Psychiatry 2000; 177: 149-55.

47 De Beurs E, Zitman FG. De Brief Symptom Inventory (BSI): de betrouwbaarheid en validiteit van een handzaam alternatief voor de SCL-90. [The Brief Symptom Inventory (BSI): reliability and validity of a feasible alternative for the SCL-90] Maandblad Geestelijke Volksgezondheid 2006; 61: 120-19.

48 Kirschbaum C, Dettenborn L, Stalder T, Foley P, Steudte S, Tietze A, et al. Cortisol in hair: a retrospective measure of cortisol levels over prolonged periods of time. Biol Psychiatry 2010; 67(9): 212S.

49 Lee DY, Kim E, Choi MH. Technical and clinical aspects of cortisol as a biochemical marker of chronic stress. BMB Rep 2015; 48(4): 209-16.

50 Noppe G, Rijke YB, Dorst K, Akker ELT, Rossum EFC. LC-MS/MS-based method for long-term steroid profiling in human scalp hair. Clin Endocrinol 2015; 83(2): $162-6$

51 First MB, Spitzer RL, Groenestijn M. Gestructureerd klinisch interview voor de vaststelling van DSM-IV As I stoornissen. [Structured Clinical Interview for DSM-IV axis I Disorders] Swets Test Publishers, 1999.

52 First MB S, Spitzer R L, Gibbon M, Williams J BW. Structured Clinical Interview for DSM-IV-TR Axis I Disorders, Research Version, Patient Edition. (SCID-I/P). Biometrics Research, New York State Psychiatric Institute, 2002.

53 Lobbestael J, Leurgans M, Arntz A. Inter-rater reliability of the Structured Clinical Interview for DSM-IV Axis I Disorders (SCID I) and Axis II Disorders (SCID II). Clin Psychol Psychother 2011; 18(1): 75-9. 
54 Derogatis L, Melisaratos N. The Brief Symptom Inventory: an introductory report. Psychol Med 1983; 13(3): 595-605.

55 Derogatis LR. BSI 18, Brief Symptom Inventory 18: Administration, Scoring and Procedures Manual. NCS Pearson, Inc., 2001.

56 Hoffman MC, D'Anna-Hernandez K, Benitez P, Ross RG, Laudenslager ML. Cortisol during human fetal life: characterization of a method for processing small quantities of newborn hair from 26 to 42 weeks gestation. Dev Psychobiol 2017; 59(1): 123-7.

57 Rippe RC, Noppe G, Windhorst DA, Tiemeier H, van Rossum EF, Jaddoe VW et al. Splitting hair for cortisol? Associations of socio-economic status, ethnicity, hair color, gender and other child characteristics with hair cortisol and cortisone. Psychoneuroendocrinology 2016; 66: 56-64.

58 Freemantle N, Marston L, Walters K, Wood J, Reynolds MR, Petersen I. Making inferences on treatment effects from real world data: propensity scores, confounding by indication, and other perils for the unwary in observational research. $B M J$ 2013; 347: f6409.

59 Altman DG. Practical statistics for medical research. Stat Med 1991; 10(10): 1635-6.

60 Grote NK, Bridge JA, Gavin AR, Melville JL, Iyengar S, Katon WJ. A metaanalysis of depression during pregnancy and the risk of preterm birth, low birth weight, and intrauterine growth restriction. Arch Gen Psychiatry 2010; 67(10): 1012-24.

61 Wei J, Sun G, Zhao L, Yang X, Liu X, Lin D, et al. Analysis of hair cortisol level in first-episodic and recurrent female patients with depression compared to healthy controls. J Affect Disord 2015; 175: 299-302.

62 Dettenborn L, Muhtz C, Skoluda N, Stalder T, Steudte S, Hinkelmann K, et al. Introducing a novel method to assess cumulative steroid concentrations: increased hair cortisol concentrations over 6 months in medicated patients with depression. Stress 2012; 15(3): 348-53.
63 Benediktsson R, Calder AA, Edwards CR, Seckl JR. Placental 11 beta-hydroxysteroid dehydrogenase: a key regulator of fetal glucocorticoid exposure. Clin Endocrinol (Oxf) 1997; 46(2): 161-6.

64 Davis M, West K, Bilms J, Morelen D, Suveg C. A systematic review of parent-child synchrony: it is more than skin deep. Dev Psychobiol 2018; 60(6): 674-91.

65 Feldman R. The neurobiology of human attachments. Trends Cogn Sci 2017; 21 (2): 80-99.

66 Kalliokoski O, Jellestad FK, Murison R. A systematic review of studies utilizing hair glucocorticoids as a measure of stress suggests the marker is more appropriate for quantifying short-term stressors. Sci Rep 2019; 9(1): 11997.

67 Wester VL, Staufenbiel SM, Veldhorst MA, Visser JA, Manenschijn L, Koper JW et al. Long-term cortisol levels measured in scalp hair of obese patients. Obesity (Silver Spring) 2014; 22(9): 1956-8.

68 Stalder T, Kirschbaum C, Alexander N, Bornstein SR, Gao W, Miller R, et al. Cortisol in hair and the metabolic syndrome. J Clin Endocrinol Metab 2013; 98(6): 2573-80.

69 Zijlmans MA, Riksen-Walraven JM, de Weerth C. Associations between maternal prenatal cortisol concentrations and child outcomes: a systematic review. Neurosci Biobehav Rev 2015; 53: 1-24.

70 Glynn LM, Davis EP, Sandman CA. New insights into the role of perinata HPA-axis dysregulation in postpartum depression. Neuropeptides 2013: 47(6): 363-70. 